\title{
Study and Analysis on Binary Offset Carrier (BOC) Modulation in Satellite Navigation Systems
}

\author{
A. Khine Myint Mon, B. Pan Thu Tun, C. Mr. Atul P. Shukla
}

\begin{abstract}
The navigation signals being used in present and future Navigation Satellite System are studied in this paper. Traditional Global Position System (GPS) is using Binary Phase shift keying (BPSK) modulation with conventional rectangular (non return to zero) spreading symbols. Modernized GPS system will be making use of new modulation technique called Binary Offset Carrier (BOC) modulation that is better modulation design for next-generation radio navigation systems. Different types of BOC signals are studied and their advantages over compared with conventional PSK modulations. The autocorrelation function (ACF) and the power spectral density (PSD) characteristics of all navigation signals and all modulations are discussed. Generation of variant of BOC signals with sine sub-carrier and cosine sub-carrier are simulated by using MATLAB.
\end{abstract}

Keywords: Binary Offset Carrier (BOC), BPSK, GPS, Modulation, Navigation Satellite System, MATLAB, power spectral density (PSD).

\section{Introduction}

Nowadays, satellite based positioning systems are widely in use. The primary purpose of these systems is to provide position, velocity and time to any user on or near the earth's surface. The user determines his position in space and time by measuring his range to at least four satellites whose position and time are accurately determined, the range being defined as the distance between the user and a satellite. The satellites of a satellite based positioning system transmit signals on several L-band carriers. The carriers are modulated by a spreading code that consists of consecutive chips, the polarity of which being determined by a pseudo-random code (PRN) and each chip of the PRN code mapping to a particular pulse. For so-called BPSK modulations, the pulse has a rectangular shape.

Each satellite uses unique PRN codes. The separation between satellites is possible because the PRN codes do not correlate with each other. The spreading codes are modulated by the navigation message that provides information about the satellite position. The times at which the satellites start sending the navigation message are synchronized.

Galileo is the European global navigation satellite system (GNSS) providing a highly accurate, guaranteed and global positioning service under civilian control.

Global Positioning System (GPS) has run successfully for many years but its positioning precision of civil signal is not high enough for user demands. Compared to GPS, Galileo can provide many services such as Open Service (OS), Public Regulated Service (PRS), Commercial Service (CS), Safety of Life (SOL) service and Safe and Rescue (SAR) service. European Galileo will come true that users will be satisfied with positioning precision of Galileo civil signal (signals will offer a guaranteed accuracy down to 1 meter, with value-added services achieving a real-time accuracy of $10 \mathrm{~cm}$ ). For that, a new modulation technology, Binary Offset Carrier (BOC), is taken by Galileo in radio navigation satellite systems.

\section{Binary Offset Modulation (BOC)}

BOC describes a class of spread-spectrum modulations recently introduced for the next generation of global navigation satellite systems. The design strategies of these BOC signals have so far focused on the spectral properties of these signals. The new generation GNSS, modernized GPS, and the European Galileo system will use BOC signals on different carriers and with different parameters. The main reasons for creating BOC signals were the need to improve traditional GNSS signals properties for better resistance to multi path 
interferences of all kinds and receiver noise and the need for improved spectral sharing of the allocated bandwidth with existing signals (single carrier GPS) or future signals of the same class.

Since Binary Phase Shift Keying (BPSK) modulation is often used in the spread spectrum satellite communication systems, the splitting spectrum character of the BOC-modulated signals can achieve the spectrum isolation with the BPSK signals in the same band. So, BOC modulation will be widely applied gradually as the crowding of the communication band.

BOC modulations offer two independent design parameters: sub-carrier frequency $\mathrm{f}_{\mathrm{s}}$ (in MHz) and spreading code rate $\mathrm{f}_{\mathrm{c}}$ (in Mcps). These two parameters provide freedom to concentrate signal power within specific parts of the allocated band to reduce interference with the reception of other signals. The result of the sub-carrier modulation is to split the classical BPSK spectrum in two symmetrical components with no remaining power on carrier frequency. The product is a symmetric split spectrum with two main lobes shifted from the carrier frequency by the amount equal to the sub-carrier frequency.

When it comes to the reception of the signal, the redundancy in the upper and lower sidebands of BOC modulations offers practical advantages in receiver processing for signal acquisition, code tracking, carrier tracking, and data demodulation. In most ways, receiver processing of BOC modulations is similar to receiver processing of PSK-R (Phase Shift Keying with Rectangular symbol) modulations. However, BOC modulations offer some unique opportunities for variations in receiver processing that can provide advantages in implementation and performance.

The best receiver performance is obtained by processing both sidebands coherently as a single signal, not only because all the signal energy is combined coherently, but also because the unique spectral shape of the dual-sideband modulation leads to better ranging performance. However, since each of the two spectral sidebands redundantly contains all the information needed for ranging and data demodulation, the distinct sidebands can be processed separately if desired. The receiver treats the BOC modulation like a PSKR modulation centered at one of the two sub-carrier frequencies. If desired, filtering can be used to select only the desired sideband and prevent aliasing at lower sampling rates. Thus, processing one sideband is identical to processing a conventional GPS signal with a PSK-R modulation.

Another motivation for sideband processing of BOC modulations is interference avoidance. Simple circuitry in a receiver can detect when partial-band interference is encountered that obscures only one sideband of the BOC modulation, and can reconfigure the receiver to process only the sideband not being interfered with. This approach can provide substantial immunity to some types of interference without the complexity of interference mitigation circuitry.

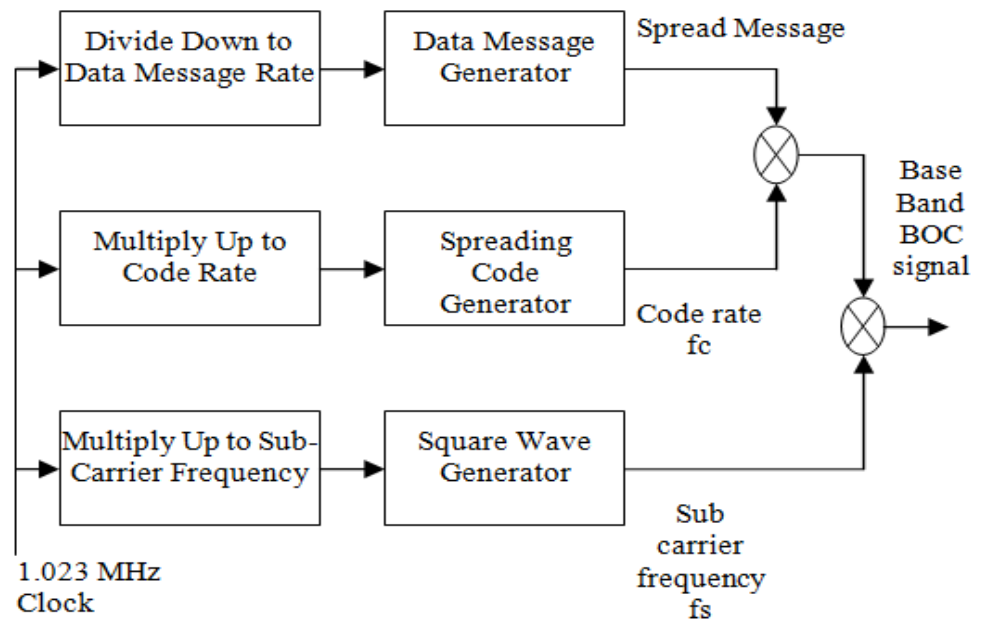

Figure (1) Block Diagram of BOC Modulation Signal Generation

Figure (1) is shown the block diagram of BOC modulation signal generation. The BOC signal consists in a three or two sets of sequences, namely pseudo random noise (PRN), square sub-carrier and data. Indeed, BOC modulation uses also a spread spectrum to achieve the direct spectrum spreading on the transmitter, then on the receiver a local PRN, which is synchronized with the one in the transmitter, is used to de-spread the received signal. BOC modulated signal is generated in two steps- Firstly a PRN sequence modulates the

DOI: $10.9790 / 2834-110501115123 \quad$ www.iosrjournals.org $\quad 116 \mid$ Page


navigation data message and then this demodulated data is multiplied by a sub-carrier having frequency $\mathrm{f}_{\mathrm{s}}$ which splits the spectrum of the signal into two parts. Generation of data, spreading code, sub-carrier, and RF uses a common clock so that zero crossings are aligned.

\section{Aim of the Study}

The main aim is to study and analyze the BOC modulated signals which can be used in Global Navigation Satellite system (GNSS) applications. The comparison of various BOC modulation signals over BPSK modulation signal is studied. Then various BOC modulation signals are generated by using MATLAB.

Other objectives are presented as follow:

$>$ To reduce the interference with BPSK modulated signal

$>$ To improve bandwidth efficiency

$>$ To be less multi path error

$>$ To lead better ranging performance

$>$ To get best receiver performance

\section{BPSK Modulation in GPS}

The Binary Phase Shift Keying (BPSK) modulation is characterized for having only two levels (binary) of symbols $\{+1,-1\}$ for bit 0 and bit 1 respectively. Its general expression in pass band can be written as follow:

$$
s(t)=A_{c} \sum_{k=-\infty}^{+\infty} \cos \left(2 \pi f t+\phi_{c}+\theta_{i, k}\right) \cdot p(t-k T)
$$

where:

$\mathrm{A}_{\mathrm{c}}$ is the amplitude.

$\phi_{\mathrm{c}}$ is the offset phase.

$\theta_{\mathrm{i}, \mathrm{k}}$ is the binary symbol $\{ \pm 1\}$.

$\mathrm{p}(\mathrm{t})$ is the shaping pulse of duration $\mathrm{T}$.

$\mathrm{k}$ index numerates symbols along time. It is taken from minus infinity to plus infinity as it is a theoretical form.

Index (i) numerates what symbol is taken from the binary alphabet $\{ \pm 1\}$ depending on $\mathrm{i}=0,1$.

GPS uses binary phase shift keying (BPSK) to modulate the codes on to the carrier. Change in code state causes a 180 degree phase shift in carrier.

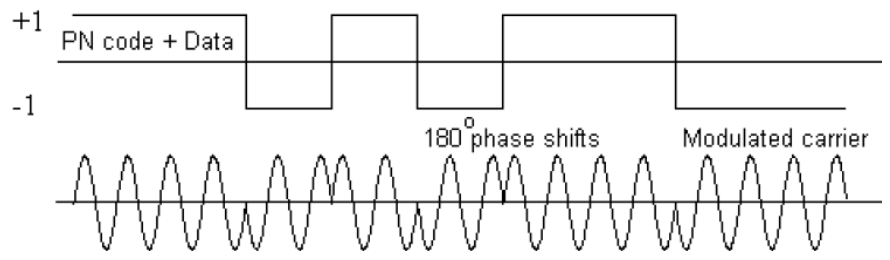

Figure (2) BPSK Modulation Scheme

GPS carrier signal is a sinusoidal signal with frequency $1575.42 \mathrm{MHz}$ (L1) and $1227.60 \mathrm{MHz}$ (L2). Each satellite transmits the same carrier frequencies at these two frequencies. These signals are designated as L1 and L2. They are coherently selected multiples of a $10.23 \mathrm{MHz}$ master clock, derived from an atomic standard.

GPS uses Code Division Multiplexing for enabling different satellites to use the same carrier frequency simultaneously. Each carrier frequency is BPSK modulated by 'Ranging Codes'. These ranging codes are different for different satellites and are orthogonal to each other. This enables the user to access the signals from different satellites at the same frequency without interference. The output transmitter power is 44.8 Watts in L1 and 4.32 Watts in L2. Figure (3) shows how to generate GPS signal by using BPSK modulation and L1 carrier. 


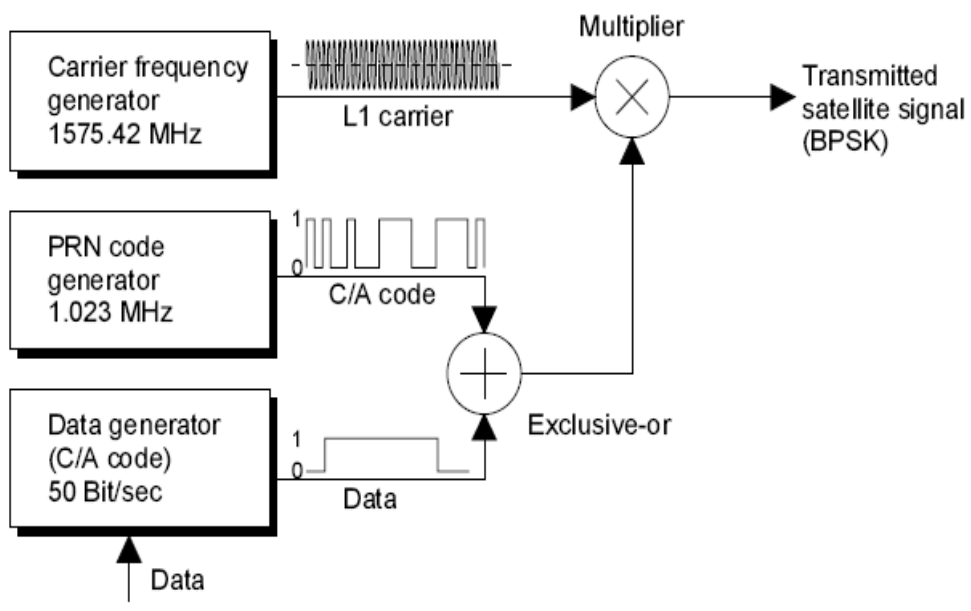

Figure (3) GPS Broadcast Signal Generation

\section{BOC Modulation for GNSS Signals}

As the time increases, the demand for improving accuracy in GNSS Systems increases. Current signaling for GPS employs phase shift keying (PSK) modulation using conventional rectangular (non-return to zero) spreading symbols. The BOC modulation is become for improved spectral sharing of the allocated bandwidth with existing signals (single carrier GPS) and to improve traditional GNSS signals properties for better resistance to multi-path interferences of all kinds and receiver noise

To reduce the effect of noise and multipath in GPS signaling structure two methods can be employed with existing modulation scheme, namely:

$>$ Employing faster keying rates

$>$ To increase the transmitted power

But bandwidth limitations preclude further improvements that might be obtained using PSK-R modulations with faster keying rates. Also, increasing transmitted power to improve accuracy is expensive and has a limited effect on multipath performance. For this purpose BOC modulation has been introduced with improved performance and implementation simplicity.

\section{A. Advantages and Disadvantages of BOC Modulation}

BOC modulation provides certain advantages over conventional PSK modulation such as:

$>$ Improved spectral sharing of the allocated bandwidth with existing signals or future signals of the same class.

$>$ Reduced mutual interference on common carrier frequencies.

Their main advantage is a better use of the spectrum, which makes the separation with GPS signals easier. In particular, such high frequency content may improve signal tracking accuracy in noisy and multi-path environments. The use of a spreading time series that incorporates BOC spreading symbols helps to incorporate such high frequency content.

A BOC spreading symbol is a higher frequency spreading symbol than a BPSK spreading symbol. BOC spreading symbols also are said to have more high frequency content than do BPSK spreading symbols since a larger percentage of total signal power of a spreading time series is located at high frequencies with BOC spreading symbols when compared to BPSK spreading symbols.

Compared with the classical BPSK modulated pseudo random (PRN) code, a BOC-modulated PRN signal has additional peaks in the autocorrelation function. The width of the main lobe of the ACF decreases (compared with BPSK, where the main lobe width is 2 chips), but additional side lobes appear in the 2-chip interval, which makes the ACF to become 'ambiguous'. 


\section{B. Definition of Binary Offset Carrier Modulation}

Binary Offset Carrier (BOC) modulation is a type of spread spectrum modulation and extension of BPSK (where there is no sub-carrier). A BOC-modulated signal is the product in the time domain of the following components:

(a) A sinusoidal carrier,

(b) A subcarrier,

(c) A pseudorandom noise (PRN) spreading code,

(d) A data sequence.

BOC modulation can be expressed as:

$\mathrm{s}(\mathrm{t})=2 \mathrm{Ad}(\mathrm{t}) \mathrm{c}(\mathrm{t}) \mathrm{s}_{\mathrm{c}}(\mathrm{t}) \cos (\omega \mathrm{t}+\theta)$

where;

A denotes the average signal power,

$d(t)$ is the navigation data signal with frequency $f_{d}$,

$c(t)$ is the PN code signal with frequency $f_{c}$, and

$s_{c}(t)$ is the sub-carrier with frequency $f_{s}$.

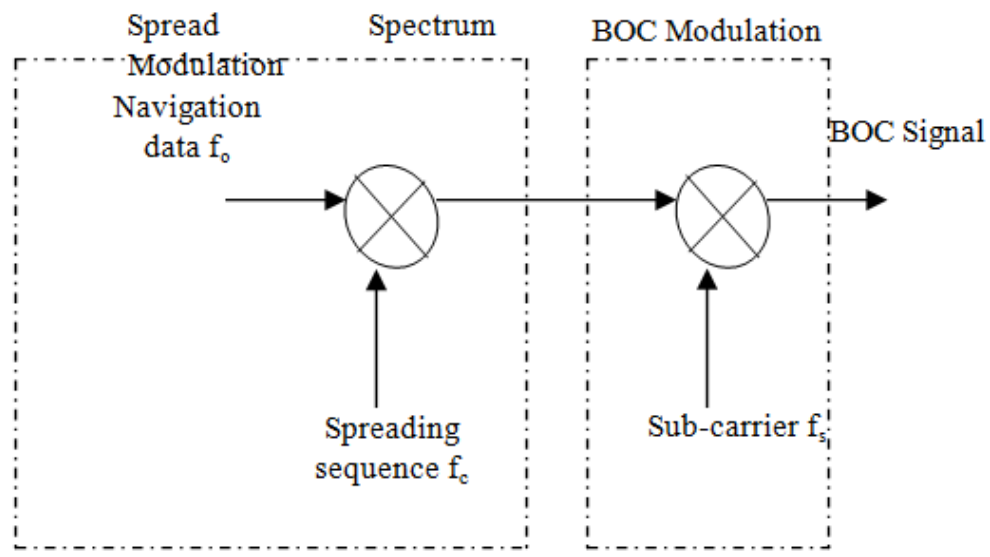

Figure (4) Typical Block Diagram of generation of BOC signal

BOC modulation currently used in Galileo is a square sub-carrier modulation, where a signal is multiplied by a rectangular sub-carrier of frequency $\mathrm{f}_{\mathrm{s}}$ equal or higher to the chip (CDMA) rate. Following this sub-carrier multiplication, the spectrum of the signal is divided into two parts, therefore BOC modulation is also known as a split-spectrum modulation.

The main idea behind BOC modulation is to reduce the interference with BPSK modulated signal, which has a sinc function shaped spectrum. Therefore, BPSK-modulated signals such as C/A GPS codes have most of their spectral energy concentrated around the carrier frequency, while BOC-modulated signals have low energy around the carrier frequency and two main spectral lobes further away from the carrier (thus, the name of split-spectrum).

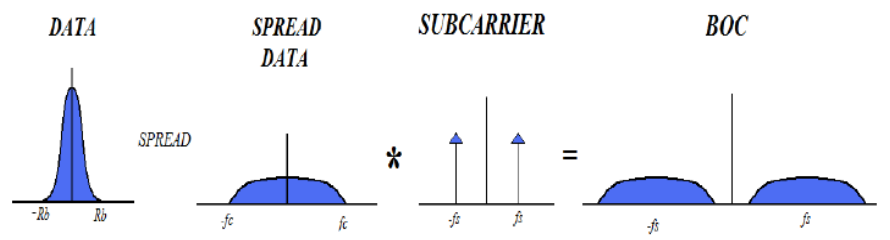

Figure (5) BOC signal

\section{Power Spectrum of BOC Modulation}

In the power spectral density, the sum of the number of main lobes and side lobes between the main lobes is equal to $\mathrm{N}$, twice the ratio of the subcarrier frequency to the code rate. As in conventional PSK, the zero crossings of each main lobe are spaced by twice the code rate, while the zero crossings of each side lobe are 
spaced at the code rate. For example, $\mathrm{N}=5$ for a $\operatorname{BOC}(5,2)$ modulation, and its spectrum has three side lobes between the two main lobes.

Maxima of the main lobes occur at frequencies somewhat below the sub-carrier frequency because of the coherent interactions between the upper and lower sidebands.

\section{Different Types of BOC Signals}

BOC modulation has several variants:

(a) Sine BOC ( $\operatorname{SinBOC)}$

(b) Cosine $\mathrm{BOC}(\mathrm{CosBOC})$

(c) Alternative BOC (AltBOC)

(d) Multiplexed BOC (MBOC)

- Composite BOC (CBOC)

- Time Multiplexed BOC (TMBOC)

(e) Double BOC (DBOC) etc. and some of them have been currently selected for Galileo GNSS signals

\section{Generation of Various BOC Signals}

In this portion, $\mathrm{BOC}(1,1)$, $\mathrm{BOC}(6,1), \mathrm{BOC}(10,5), \mathrm{BOC}(15,2.5)$ and $\mathrm{BOC}(5,2)$ with both sine sub-carrier and cosine sub-carrier are generated by using MATLAB.

When simulation is being done, virtual memory need to enough to run the program quickly. MATLAB stores all the variables generated when a script is executed and the longer the vectors the larger is the space used in memory. So, the problem with the memory comes if the sampling frequency used is very high. So, the program runs quickly out of RAM memory if that issue is not taken into account.

The aim of these power spectrum density (PSD) plots is to give the information how these modulations are such as peak amplitude and side-lobes power. BOC modulation can be presented as BOC (fs, $\mathrm{fc}$ ) and BOC $(\mathrm{m}, \mathrm{n})$.

$$
\begin{aligned}
& f_{s}=\frac{1}{2 T_{s}} \\
& f_{c}=\frac{2 f_{s}}{N}=\frac{1}{N T_{s}} \\
& \mathrm{~N}=\frac{2 \mathrm{f}_{\mathrm{s}}}{\mathrm{f}_{\mathrm{c}}}
\end{aligned}
$$

where;

$\mathrm{f}_{\mathrm{s}}$ is subcarrier frequency $(\mathrm{MHz})=\mathrm{m} * \mathrm{f}_{\text {ref }}$

$\mathrm{f}_{\mathrm{c}}$ is the spreading code rate $(\mathrm{Mcps})=\mathrm{n} * \mathrm{f}_{\text {ref }}$ $\mathrm{f}_{\text {ref }}$ is reference frequency $=1.023 \mathrm{MHz}$

The sum of main lobes and side lobes between the main lobes is equal to $\mathrm{N}$, twice the ratio of the subcarrier frequency $\left(f_{s}\right)$ to the code rate $\left(f_{c}\right)$.

\section{A. $\operatorname{BOC}(1,1)$ Modulation}

\section{Simulation Results}

The $\operatorname{BOC}(1,1)$ is the simplest representation of a PSD function as shown in figure (6).

For the $\mathrm{BOC}(1,1)$ : fs $=1.023 \mathrm{MHz}, \mathrm{fc}=1.023 \mathrm{MHz}$

Therefore $\mathrm{N}=2$ is the BOC modulation order for BOCsin $(1,1)$ signal.

The frequency of the maximum PSD from a cosine sub-carrier is higher than that of the maximum PSD from a sine sub-carrier. PSD with sine sub-carrier spread more power on main lobes whereas PSD cosine sub-carriers spread more power in side lobes. BOC $(1,1)$ power is similar to BPSK $(1)$. 


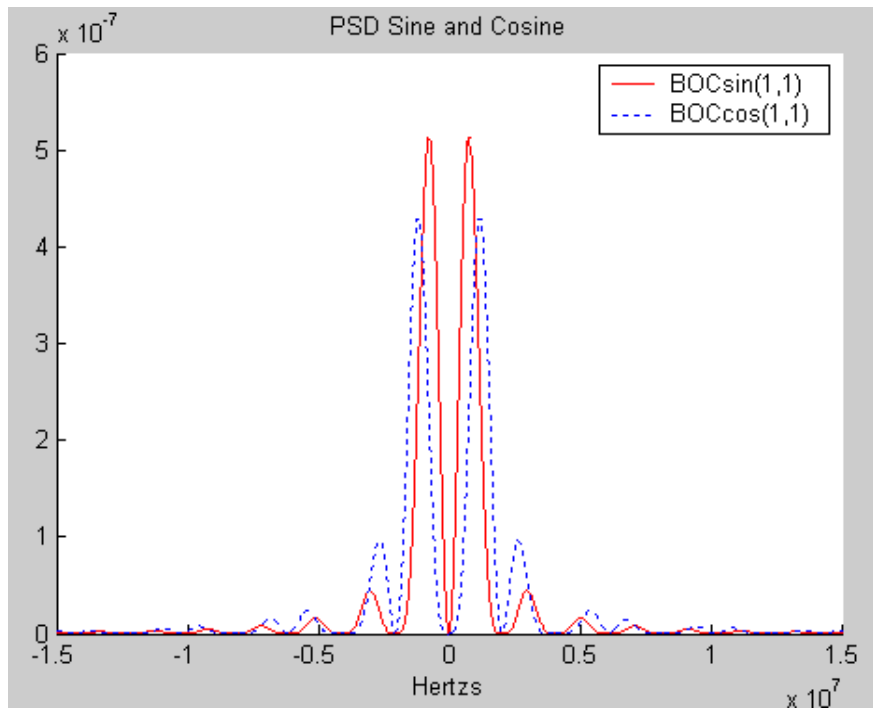

Figure (6) MATLAB simulated PSD function for $\operatorname{BOCsin}(1,1)$ and $B O C \cos (1,1)$

\section{B. $B O C(6,1)$ Modulation}

The weighted sum of $\operatorname{BOC}(1,1)$ and $\operatorname{BOC}(6,1)$ signal is produced to achieve MBOC signal spectrum.

For the $\operatorname{BOC}(6,1)$ :

$\mathrm{fs}=6 * 1.023=6.138 \mathrm{MHz}$,

$\mathrm{fc}=1.023 \mathrm{MHz}$

Therefore $\mathrm{N}=12$ is the BOC modulation order for BOCsin $(6,1)$ signal. The PSD function of the BOC $(6,1)$ by using sine sub-carrier and cosine sub-carrier are shown in figure (7).

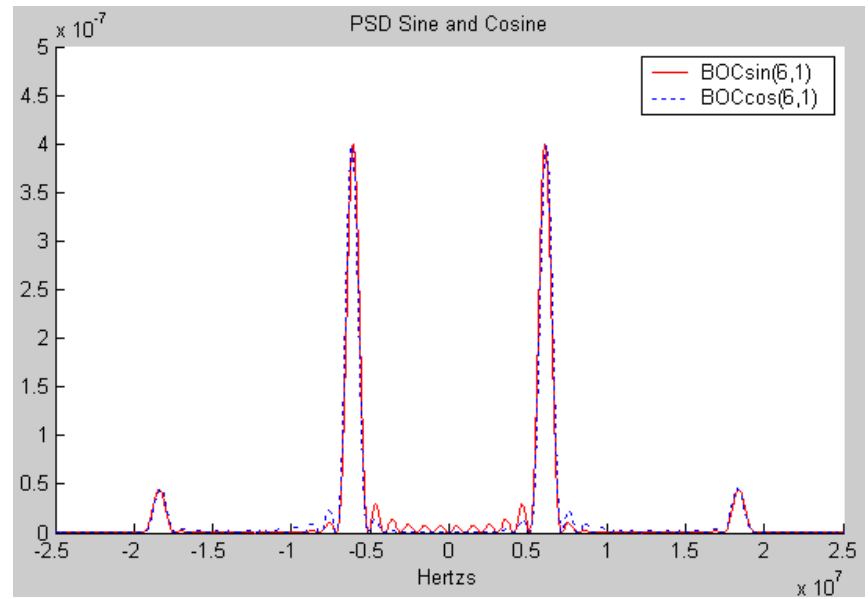

Figure (7) MAT LAB simulated PSD function for BOCsin $(6,1)$ and $\mathrm{BOC} \cos (6,1)$

\section{BOC (10, 5) Modulation}

The BOC $(10,5)$ modulation was designed to allow the new military signal to be transmitted at much higher power while minimizing interference with existing signals and providing many other benefits, ranging from implementation ease to performance. The narrower peak and multimode structure of the BOC (10, 5) PSD function are illustrated in figure (8).

For the BOC $(10,5)$ :

fs $=10 * 1.023=10.23 \mathrm{MHz}$,

$\mathrm{fc}=5 * 1.023=5.115 \mathrm{MHz}$

Therefore $\mathrm{N}=4$ is the BOC modulation order for BOCsin $(10,5)$ signal. 


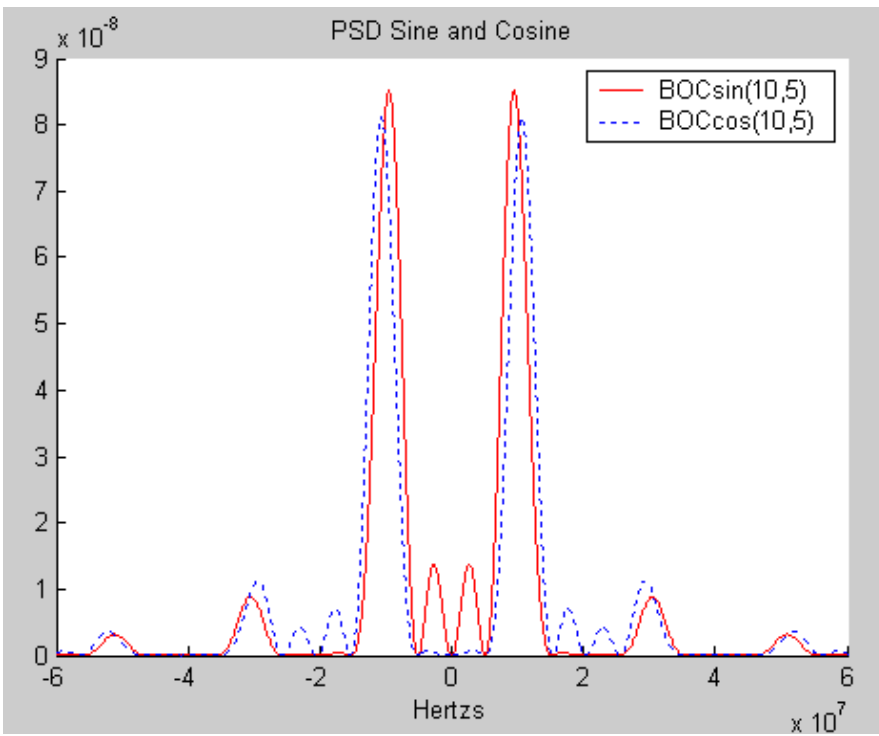

Figure (8) MATLAB simulated PSD function for BOCsin $(10,5)$ and BOCcos $(10,5)$

\section{D. $\operatorname{BOC}(15,2.5)$ Modulation}

Type of BOC $(15,2.5)$ signal is intended for specific services such as the Galileo public regulated service (PRS) to be of interest for experts.

For the BOC $(15,2.5)$ :

$\mathrm{fs}=15 * 1.023=15.345 \mathrm{MHz}$,

$\mathrm{fc}=2.5 * 1.023=2.5575 \mathrm{MHz}$

Therefore $\mathrm{N}=12$ is the BOC modulation order for BOCsin $(15,2.5)$ signal.

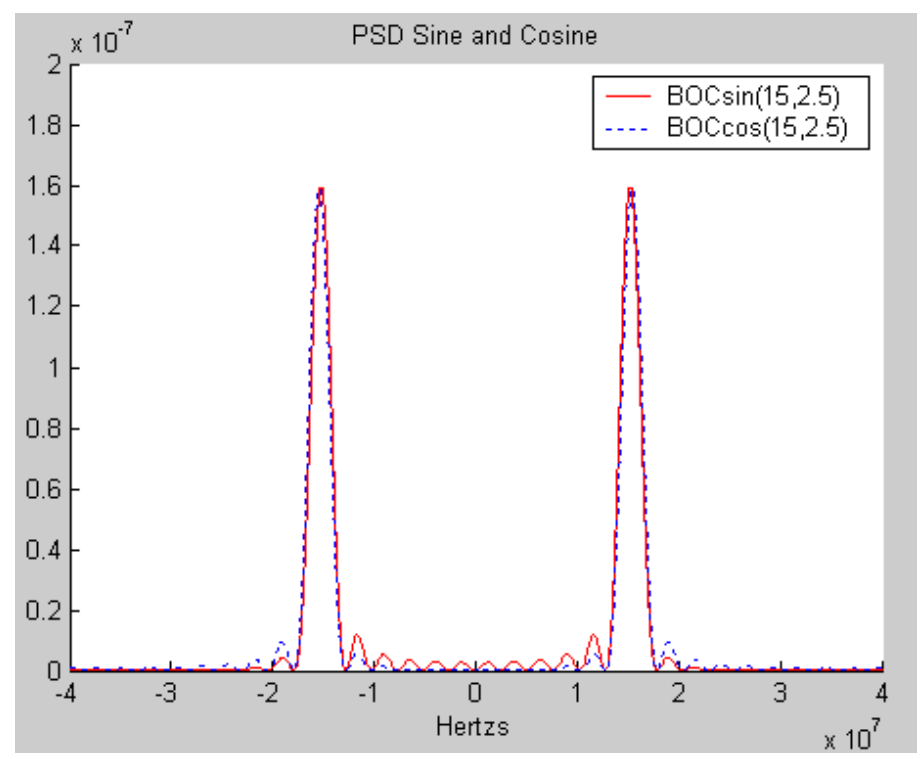

Figure (9) MATLAB simulated PSD function for BOCsin $(15,2.5)$ and BOCcos $(15,2.5)$

\section{Conclusion and Further Study}

In this paper, various Navigation Satellite Systems like GPS launched by US, GLONASS launched by Russia, GALILEO launched by Europe, COMPASS launched by China and IRNSS launched by India are studied. Differences of their number of satellite, frequencies, applications, services and modulation techniques are studied and described. 
Especially Binary Offset Carrier (BOC) modulation is described with its definition, spectral analysis, properties, advantages and disadvantages, signal structure. Power spectrum density (PSD), auto correlation function $(\mathrm{ACF})$ and various types of BOC signal are presented. Comparison of BOC and BPSK modulations are analyzed. And then how to generate the BOC signals are studied.

By using MATLAB, studying and simulation of generation of BOC $(1,1)$ BOC $(6,1)$, BOC $(10,5)$, and BOC $(15,2.5)$ modulated signals with both sine sub-carrier and cosine sub-carrier has been done in this project.

The study done could be extended by the following points:

$>$ Generation of various BOC signals by using MATLAB

$>$ Simulation of different acquisition algorithms and Tracking Methods for BOC signals

$>$ Considering the effects of BOC modulation for the best performance and low cost

$>$ Comparison of Spectral and correlation properties of various BOC modulated signals

$>$ Performance comparison of various method

$>$ Acquisition performance

$>$ Tracking performance

$>$ Noise performance

$>$ Effect of Multipath

\section{Acknowledgement}

Firstly I would like to thank my parents, sister and brother for their best wishes to me and their complete support. Special thanks are due to my supervisor: Mr. Atul P. Shukla, Space Application Center in Ahmedabad, India and my friend: Dr. Pan Thu Tun, Associate Professor and Head of Department of Electronic Engineering, Technological University (Kyaukse), Mandalay Division, Myanmar for their kindness of guidelines for this paper. I greatly would like to express my thanks to my friends and all persons whom will concern to support in preparing and sending this paper.

\section{References}

[1]. "Binary Offset Carrier Modulations for Radio navigation", JOHN W. BETZ, The MITRE Corporation, Bedford, Massachusetts.

[2]. "Galileo Signal Generation Simulation Analysis", Roger Canalda Pedrós, Department of Computer and Electronic Engineering, University of Limerick.

[3]. "Galileo Open Service, Signal In Space, Interface Control Document OS SIS ICD, Draft 1", European Space Agency/European GNSS Supervisory Authority.

[4]. "Galileo signal design: State of Art", Umberto Robustelli, Salvatore Gaglione,Raffaele Santamaria ,Mario Vultaggio.

[5]. "Performance of Galileo L1 Signal Candidates", Gunter W. Hein, Markus Irsigler, Jose Angel Avila Rodriguez and Thomas Pany.

[6]. "Satellite Navigation", Rajat Acharya.

[7]. "Galileo navigation signals and frequencies", European Space Agency.

[8]. “Galileo Signal-In-Space Design”, Ester Armengou Miret.

[9]. www.esa.int/esaNA/galileo.html

[10]. www.insidegnss.com

[11]. www.esa.int/esaNA/galileo.html

[12]. www.en.wikipedia.org/wiki.html 